\title{
Distances between quantum states in the tomographic-probability representation
}

\author{
S N Filippov' and V I Man'ko \\ ${ }^{1}$ Moscow Institute of Physics and Technology, Moscow, Russia \\ 2 P N Lebedev Physical Institute, Moscow, Russia \\ E-mail: filippovsn@gmail.com, manko@sci.lebedev.ru
}

\begin{abstract}
Distances between quantum states are reviewed within the framework of the tomographic-probability representation. Tomographic approach is based on observed probabilities and is straightforward for data processing. Different states are distinguished by comparing corresponding probability-distribution functions. Fidelity as well as other distance measures are expressed in terms of tomograms.

PACS numbers: 03.65.Ta, 03.65.Wj, 03.67.-a
\end{abstract}




\section{Introduction}

Comparing quantum states is of crucial importance for the theory of quantum information processing. At any step of computation one should be aware of a system state and know how much it deviates from desired evolution. Moreover, it is necessary to compare different states and quantify the distance between them by virtue of experimental data. Measurements enable us to inquire some information about system but such an information is presented in the form of observed probabilities. For this reason one needs a way of comparing quantum states with the help of measured probability distributions and try not to employ the density matrix formalism.

Probability-distribution functions enable one to reconstruct quantum states [1, 2, 3, 4, 5]. On the other hand, quantum states can be identified with observed probability distributions rather than density operators or Wigner functions [6, 7]. According to this representation, quantum states are associated with fair probability-distribution functions called tomograms. The tomographic representation of quantum states with continuous variables (position, momentum) is introduced in [1] and that of states with discrete variables (spins) is introduced in [4, 5]. Such an approach describes not only states but can also be developed to build the whole tomographic picture of quantum mechanics (see the reviews [8, 9]).

In this paper, we use peculiarities of the tomographic-probability representation to express distances between spin and light states in terms of quantum tomograms. The main idea of this consideration is that two states are close to each other if the corresponding probability distributions differ slightly, and are far apart from each other if their tomograms do not match significantly. These basic ideas were outlined and successfully applied to Fock's, coherent, squeezed, and Schrödinger cat states in [10, 11].

The paper is organized as follows.

In Section 2, we are aimed at recalling the tomographic representation of spin and light states and observables. Star product of tomographic symbols is also introduced in concise manner. In Section 3, we consider conventional distance measures which are often used in quantum information theory. Tomographic analogues of these distances are introduced for spins (qubits, qudits) in Section 3.1 and for photon states in Section 3.2. In Section 4, conclusions and prospects are presented.

\section{State tomograms}

To begin with, whatever system is under investigation (spin, qubit, qudit, light, particle) it can be associated with tomographic symbol of the form

$$
w(\mathbf{x})=\operatorname{Tr}(\hat{\rho} \hat{U}(\mathbf{x}))
$$

where $\hat{\rho}$ is the density operator and $\hat{U}(\mathbf{x})$ is a dequantizer operator depending on a particular set of parameters $\mathbf{x}$. 
The inverse mapping of tomographic symbols onto density operators is

$$
\hat{\rho}=\int \mathrm{d} \mathbf{x} w(\mathbf{x}) \hat{D}(\mathbf{x}),
$$

where $\hat{D}(\mathbf{x})$ is a quantizer operator.

If operator $\hat{A}$ is given, it is also possible to construct tomographic symbol of this operator. The only thing one should do is to replace density operator $\hat{\rho}$ by $\hat{A}$. If one knows symbols $w_{A}(\mathbf{x})$ and $w_{B}(\mathbf{x})$ of operators $\hat{A}$ and $\hat{B}$, respectively, then the symbol

of operator $\hat{A} \hat{B}$ is equal to the star-product of separate tomographic symbols. Namely,

$$
\begin{aligned}
w_{A B}(\mathbf{x}) & \equiv\left(w_{A} \star w_{B}\right)(\mathbf{x}) \\
& =\int w_{A}\left(\mathbf{x}_{1}\right) w_{B}\left(\mathbf{x}_{2}\right) K\left(\mathbf{x}, \mathbf{x}_{1}, \mathbf{x}_{2}\right) \mathrm{d} \mathbf{x},
\end{aligned}
$$

where $K\left(\mathbf{x}, \mathbf{x}_{1}, \mathbf{x}_{2}\right)$ is called tomographic star-product kernel. General and specific tomographic star-product schemes are discussed in [12, 13, 14, 15]. The problem of spin tomographic kernels for spins was attacked from different perspectives, e.g., in [16, 17, 18].

Particular form of quantizer and dequantizer operators depends on the system in question. Further we epitomize these operators for spin and light systems.

\subsection{Spin tomography}

By convention, basis states are eigenvectors $|j m\rangle$ of angular momentum operators $\hat{J}_{z}$ and $\hat{\mathbf{J}}^{2}$. Let $u$ be an element of group $S U(2)$ or $S U(N)$ with $N=2 j+1$. We use the following notation:

$$
\begin{aligned}
& \mathbf{x}=(m, u) \\
& \int \mathrm{d} \mathbf{x}=\sum_{m=-j}^{j} \frac{1}{8 \pi^{2}} \int_{0}^{2 \pi} \mathrm{d} \alpha \int_{0}^{\pi} \sin \beta \mathrm{d} \beta \int_{0}^{2 \pi} \mathrm{d} \gamma,
\end{aligned}
$$

where the latter equation implies that $u \in S U(2)$ is parametrized by Euler angles $\alpha, \beta$, and $\gamma$. Then scanning and reconstruction procedures of spin tomography are given by dequantizer and quantizer operators of the form [17]:

$$
\begin{aligned}
\hat{U}(\mathbf{x})=u^{\dagger}|m\rangle\langle m| u, & \\
\hat{D}(\mathbf{x})=(2 j+1)[\hat{U}(\mathbf{x}) & -\frac{1}{2} \hat{R}_{+}(u) \hat{U}(\mathbf{x}) \hat{R}_{-}(u) \\
& \left.-\frac{1}{2} \hat{R}_{-}(u) \hat{U}(\mathbf{x}) \hat{R}_{+}(u)\right],
\end{aligned}
$$

where 


$$
\begin{aligned}
& \hat{R}_{+}(u)=\sum_{m=-j}^{j-1} u^{\dagger}|j, m+1\rangle\langle j, m| u, \\
& \hat{R}_{-}(u)=\sum_{m=-j+1}^{j} u^{\dagger}|j, m-1\rangle\langle j, m| u .
\end{aligned}
$$

\subsection{Photon-number tomography}

Quantum state of light is uniquely determined by the photon-number tomogram. In this case Fock states $|n\rangle$ form a basis, displacement operator $\hat{\mathcal{D}}(\alpha)=\exp \left[\alpha \hat{a}^{\dagger}-\alpha^{*} \hat{a}\right]$ plays role of unitary matrix in the spin tomography. In other words, the notation is

$$
\mathbf{x}=(n, \alpha), \quad \int \mathrm{d} \mathbf{x}=\sum_{n=0}^{\infty} \iint \frac{\mathrm{d}^{2} \alpha}{\pi},
$$

and tomographic procedures are given by operators [19, 20, 21, 22]

$$
\begin{aligned}
& \hat{U}(\mathbf{x})=\hat{\mathcal{D}}^{\dagger}(\alpha)|n\rangle\langle n| \hat{\mathcal{D}}(\alpha), \\
& \hat{D}(\mathbf{x})=\frac{4}{1-s^{2}}\left(\frac{s-1}{s+1}\right)^{\left(\hat{a}^{\dagger}+\alpha^{*}\right)(\hat{a}+\alpha)-n},
\end{aligned}
$$

where $s$ is an arbitrary ordering parameter [23].

\section{Distances between states in view of their tomograms}

The extent to which quantum states are similar to each other is usually expressed in terms of their density operators $\hat{\rho}_{1}$ and $\hat{\rho}_{2}$. On the other hand, unlike tomogram density matrix is not observed directly in experiment. This fact makes reasonable to express basic distance measures between states in terms of their tomograms. In this paper, we are going to explore the following standard quantities (see, e.g., [24, 25, 26, 27, 28]):

(i) Hilbert-Schmidt distance $\left\|\rho_{1}-\rho_{2}\right\|_{\mathrm{HS}} \equiv\left[\frac{1}{2} \operatorname{Tr}\left(\rho_{1}-\rho_{2}\right)^{2}\right]^{1 / 2}$;

(ii) trace distance $\frac{1}{2} \operatorname{Tr}\left|\rho_{1}-\rho_{2}\right|$, where $|A| \equiv \sqrt{A^{\dagger} A}$;

(iii) fidelity $F\left(\rho_{1}, \rho_{2}\right)=\operatorname{Tr}\left[\sqrt{\rho_{1}} \rho_{2} \sqrt{\rho_{1}}\right]^{1 / 2}$;

(iv) operator norm $\left\|\rho_{1}-\rho_{2}\right\|=\sup _{\|\psi\|=1}\left\|\left(\rho_{1}-\rho_{2}\right) \psi\right\|$.

If the reconstruction procedure is given, one is tempted to use quantizer operator $\hat{D}(\mathbf{x})$ instead of density operator whenever it is possible. As far as distance measures are concerned, such a method does not give us any advantage as compared to density operators. Here we develop an alternative approach which is based on probabilitydistribution functions only, is straightforward for computation and sheds some light on relation between measures for classical and quantum information. 
Both spin and photon number tomogram can be considered as probability vectors due to normalization conditions $\sum_{m=-j}^{j} w(m, u)=1$ and $\sum_{n=0}^{\infty} w(n, \alpha)=1$. Indeed, splitting different numbers $m$ and $n$, we obtain

$$
\begin{aligned}
& \vec{w}(u)=\left(\begin{array}{llll}
w(j, u) & w(j-1, u) & \cdots & w(-j, u)
\end{array}\right)^{\operatorname{tr}} \\
& \vec{w}(\alpha)=\left(\begin{array}{llll}
w(0, \alpha) & w(1, \alpha) & w(2, \alpha) & \cdots
\end{array}\right)^{\operatorname{tr}}
\end{aligned}
$$

We will discuss spin systems (qubits, qudits) first and then we will also pay attention to the photon-number tomogram.

\subsection{Distances in terms of spin tomograms}

In the paper [29], it is shown that the Hilbert-Schmidt distance between qubit $(j=1 / 2)$ states is equal to the maximal possible Euclidean distance between corresponding tomographic-probability vectors with respect to rotations in Hilbert space. Here, we extend this claim to higher dimensions.

Proposition. For an arbitrary qudit states $\rho_{1}$ and $\rho_{2}$ the following relation takes place $(N=2 j+1)$ :

$$
\begin{aligned}
& \left\|\rho_{1}-\rho_{2}\right\|_{\mathrm{HS}} \\
& =\max _{u \in S U(N)}\left[\frac{1}{2} \sum_{m=-j}^{j}\left(w_{1}(m, u)-w_{2}(m, u)\right)^{2}\right]^{1 / 2} .
\end{aligned}
$$

\section{Proof.}

Tomogram of any Hermitian operator $\hat{A}$ can be rewritten in terms of its eigenvalues $A_{l}, l=1, \ldots, N$ and unitary matrix $u_{A}$ composed of its eigenvectors as follows [9]:

$\vec{w}_{A}(u)=\left(\begin{array}{ccc}\left|\left(u u_{A}\right)_{11}\right|^{2} & \ldots & \left|\left(u u_{A}\right)_{1 N}\right|^{2} \\ \ldots & \ldots & \ldots \\ \left|\left(u u_{A}\right)_{N 1}\right|^{2} & \ldots & \left|\left(u u_{A}\right)_{N N}\right|^{2}\end{array}\right)\left(\begin{array}{c}A_{1} \\ \ldots \\ A_{N}\end{array}\right)$.

By $M$ denote $N \times N$ matrix in (16). Note that $M$ is bistochastic since all rows and all columns sum to 1 . It means that each component of vector $\vec{w}_{A}(u)$ is a convex sum of eigenvalues $A_{k}$. Moreover, sum of all components of vector $\vec{w}_{A}(u)$ equals sum of $A_{k}$. Due to these facts the maximal value of quantity

$$
\left(\vec{w}_{A}(u), \vec{w}_{A}(u)\right) \equiv \sum_{k=1}^{N} w_{A k}^{2}(u)=\sum_{k=1}^{N}\left(\sum_{l=1}^{N} M_{k l} A_{l}\right)^{2}
$$

is achieved when $M$ is identity matrix or, equivalently, $u=u_{A}^{\dagger}$. In other words, 


$$
\begin{aligned}
\max _{u \in S U(N)}\left(\vec{w}_{A}(u), \vec{w}_{A}(u)\right) & =\left(\vec{w}_{A}\left(u_{A}\right), \vec{w}_{A}\left(u_{A}\right)\right) \\
& =\sum_{k=1}^{N} A_{k}^{2}=\operatorname{Tr} A^{2}
\end{aligned}
$$

If we replace $A$ by $\left(\rho_{1}-\rho_{2}\right)$ and recall $\left\|\rho_{1}-\rho_{2}\right\|_{\mathrm{HS}}=\left[\frac{1}{2} \operatorname{Tr}\left(\rho_{1}-\rho_{2}\right)^{2}\right]^{1 / 2}$, we obtain the statement of the proposition.

In addition to this relation it is worth emphasizing that rotations by elements $u \in S U(2)$ result in smaller distance

$$
\begin{aligned}
0 & \leq \max _{u \in S U(2)}\left[\frac{1}{2} \sum_{m=-j}^{j}\left(w_{1}(m, u)-w_{2}(m, u)\right)^{2}\right]^{1 / 2} \\
& \leq\left\|\rho_{1}-\rho_{2}\right\|_{\mathrm{HS}}
\end{aligned}
$$

however, it can also serve as measure of distance because left inequality becomes equality iff $\rho_{1} \equiv \rho_{2}$.

Proposition. Trace distance between spin states $\rho_{1}$ and $\rho_{2}$ is expressed in terms of tomograms as follows:

$$
\begin{aligned}
& \frac{1}{2} \operatorname{Tr}\left|\rho_{1}-\rho_{2}\right| \\
& =\max _{u \in S U(N)}\left[\frac{1}{2} \sum_{m=-j}^{j}\left|w_{1}(m, u)-w_{2}(m, u)\right|\right]
\end{aligned}
$$

Proof. Arguing as above, we obtain the similar equation for operator $A=\rho_{1}-\rho_{2}$. Namely,

$$
\sum_{k=1}^{N}\left|w_{A k}(u)\right|=\sum_{k=1}^{N}\left|\sum_{l=1}^{N} M_{k l} A_{l}\right| .
$$

This function of variables $M_{k l}$ has the same properties as function (17) and is harmonic. For these reasons it also achieves its maximal value at the boundary determined by bistochastic matrix $M$, with the maximum being equal to

$$
\sum_{k=1}^{N}\left|A_{k}\right|=\operatorname{Tr}|A| \text {. }
$$

In other words, trace distance is equal to the maximal possible Kolmogorov distance between tomographic-probability distributions. Note that maximum is attained by the same element $\tilde{u}$ of $S U(N)$ as in case of Hilbert-Schmidt distance. 
Let us now consider fidelity.

Proposition. Tomographic-probability version of fidelity reads

$$
\begin{aligned}
& \operatorname{Tr}\left[\sqrt{\rho_{1}} \rho_{2} \sqrt{\rho_{1}}\right]^{1 / 2} \\
& =\min _{u \in S U(N)}\left[\sum_{m=-j}^{j} \sqrt{w_{1}(m, u) w_{2}(m, u)}\right]
\end{aligned}
$$

Proof. To prove this Proposition one can follow, step by step, proof of the known formula (see, e.g., [25])

$$
F\left(\rho_{1}, \rho_{2}\right)=\min _{\left\{E_{k}\right\}} \sum_{k} \sqrt{\operatorname{Tr}\left(\rho_{1} E_{k}\right) \operatorname{Tr}\left(\rho_{2} E_{k}\right)},
$$

where minimum is over all positive operator valued measures $\left\{E_{k}\right\}$. To prove that the minimum is achieved the authors [25] use effects $E_{k}$ in the form of projectors $\left|\varphi_{k}\right\rangle\left\langle\varphi_{k}\right|$. In our case we can employ $E_{m}=\sqrt{E_{m}}=u|m\rangle\langle m| u^{\dagger}$ with matrix $u$ such that $\left|\varphi_{k}\right\rangle=u|k\rangle$.

It is worth noting that fidelity is the minimal value of Bhattacharyya coefficient [30] (see also the review [28]) of two tomographic-probability distributions.

Another way to consider fidelity is to use symmetric form $F=\operatorname{Tr}\left|\sqrt{\rho_{1}} \sqrt{\rho_{2}}\right|$ and tomographic symbols of operators $\sqrt{\rho_{1}}$ and $\sqrt{\rho_{2}}$. The question arises itself how to express tomogram $w_{\sqrt{\rho}}(m, u)$ of positive operator $\sqrt{\rho}$ if we know tomogram of $w_{\rho}(m, u)$ of state $\rho$. Using spectral decomposition of density operator

$$
\rho=\sum_{m^{\prime}=-j}^{j} \rho_{m^{\prime}} u_{\rho}\left|m^{\prime}\right\rangle\left\langle m^{\prime}\right| u_{\rho}^{\dagger}
$$

it is easy to express tomogram

$w_{\rho}(m, u)=\left\langle m\left|u \rho u^{\dagger}\right| m\right\rangle=\sum_{m^{\prime}=-j}^{j} \rho_{m^{\prime}}\left|\left\langle m\left|u u_{\rho}\right| m^{\prime}\right\rangle\right|^{2}$.

From this it follows that $\rho_{m^{\prime}}=w_{\rho}\left(m^{\prime}, u_{\rho}^{\dagger}\right)$. Spectral decomposition of operator $\sqrt{\rho}$ is obtained from (25) by replacing $\rho_{m^{\prime}}$ by $\sqrt{\rho_{m^{\prime}}}$. Then we have

$w_{\sqrt{\rho}}(m, u)=\sum_{m^{\prime}=-j}^{j} \sqrt{w_{\rho}\left(m^{\prime}, u_{\rho}^{\dagger}\right)}\left|\left\langle m\left|u u_{\rho}\right| m^{\prime}\right\rangle\right|^{2}$.

Tomographic symbol of operator $\sqrt{\rho_{1}} \sqrt{\rho_{2}}$ is the star product of corresponding symbols. Kernel of this star-product for qudits is calculated, e.g., in [16, 18]. Taking advantage of (20) we obtain

$$
\operatorname{Tr}\left|\sqrt{\rho_{1}} \sqrt{\rho_{2}}\right|=\max _{u \in S U(N)}\left[\sum_{m=-j}^{j}\left|\left(w_{\sqrt{\rho_{1}}} \star w_{\sqrt{\rho_{2}}}\right)(m, u)\right|\right]
$$

If we compare (24) and (28), we reveal new properties of tomograms. In fact, 


$$
\begin{aligned}
& \min _{u \in S U(N)}\left[\sum_{m=-j}^{j} \sqrt{w_{\rho_{1}}(m, u) w_{\rho_{2}}(m, u)}\right] \\
= & \max _{u \in S U(N)}\left[\sum_{m=-j}^{j}\left|\left(w_{\sqrt{\rho_{1}}} \star w_{\sqrt{\rho_{2}}}\right)(m, u)\right|\right]
\end{aligned}
$$

As far as operator norm $\left\|\rho_{1}-\rho_{2}\right\|$ is concerned, it is equal to the maximal eigenvalue of operator $\left|\rho_{1}-\rho_{2}\right|$. Consequently, this norm is

$\left\|\rho_{1}-\rho_{2}\right\|=\max _{m=-j, \ldots, j ; u \in S U(N)}\left|w_{1}(m, u)-w_{2}(m, u)\right|$.

\subsection{Distances in terms of photon number tomograms}

Using analogy of spin and photon number tomogram, one can readily extend these results to the Hilbert-Schmidt distance between light states. For instance, HilbertSchmidt distance is

$\left\|\rho_{1}-\rho_{2}\right\|_{\mathrm{HS}}=\max _{\mathcal{D} \in S U(\infty)}\left[\frac{1}{2} \sum_{n=0}^{\infty}\left\langle n\left|\mathcal{D}\left(\rho_{1}-\rho_{2}\right) \mathcal{D}^{\dagger}\right| n\right\rangle^{2}\right]^{1 / 2}$.

So as not to resort to group $S U(\infty)$ we will only formulate inequalities in term of conventional photon number tomograms:

$$
\begin{aligned}
0 & \leq \max _{\alpha \in \mathbb{C}}\left[\frac{1}{2} \sum_{n=0}^{\infty}\left(w_{1}(n, \alpha)-w_{2}(n, \alpha)\right)^{2}\right]^{1 / 2} \\
& \leq\left\|\rho_{1}-\rho_{2}\right\|_{\mathrm{HS}} \\
0 & \leq \max _{\alpha \in \mathbb{C}}\left[\frac{1}{2} \sum_{n=0}^{\infty}\left|w_{1}(n, \alpha)-w_{2}(n, \alpha)\right| \leq \frac{1}{2} \operatorname{Tr}\left|\rho_{1}-\rho_{2}\right|,\right. \\
0 & \leq F\left(\rho_{1}, \rho_{2}\right) \leq \min _{\alpha \in \mathbb{C}}\left[\sum_{n=0}^{\infty} \sqrt{w_{1}(n, \alpha) w_{2}(n, \alpha)}\right] \\
0 & \leq \max _{n \in\{0\} \cup \mathbb{N}, \alpha \in \mathbb{C}}\left|w_{1}(n, \alpha)-w_{2}(n, \alpha)\right| \leq\left\|\rho_{1}-\rho_{2}\right\| .
\end{aligned}
$$

\section{Conclusions}

To conclude we summarize the main results of the paper.

Conventional distance measures between quantum states and fidelity, which are usually formulated for density matrices, are expressed in terms of quantum tomograms. It is demonstrated that Hilbert-Schmidt distance is related to maximal Euclidean distance of tomographic-probability vectors, trace distance is related to maximal Kolmogorov distance of tomograms, fidelity is related to minimal Bhattacharyya 
coefficient, and operator norm is related to maximum of residual tomographic symbol. Analyzing photon number tomography, these results are also extended to the case of infinite Hilbert space of Fock states and formulated in the form of inequalities. We believe the introduced quantities to be used as an alternative to distance measures based on density matrices. Interesting problem for further consideration is to develop analogues approach for continuous variables quantum systems.

\section{Acknowledgments}

This study was partially supported by the Russian Foundation for Basic Research under Project Nos. 07-02-00598, 08-02-90300, and 09-02-00142. SNF thanks the Ministry of Education and Science of the Russian Federation and the Federal Education Agency for support under Project No. 2.1.1/5909. The authors are grateful to the Organizers of the Sixteenth Central European Workshop on Quantum Optics (Turku, Finland, May 23-27, 2009) for invitation and kind hospitality. SNF would like to express his gratitude to the Organizing Committee of the Conference and especially to Professor Kalle-Antti Suominen for financial support. SNF thanks the Russian Foundation for Basic Research for travel grant No. 09-02-09240.

\section{References}

[1] Mancini S, Man'ko V I and Tombesi P 1996 Phys. Lett. A 2131

[2] Bertrand J and Bertrand P 1987 Found. Phys. 17397

[3] Vogel K and Risken H 1989 Phys. Rev. A 402847

[4] Dodonov V V and Man'ko V I 1997 Phys. Lett. A 229335

[5] Man'ko V I and Man'ko O V 1997 J. Exp. Theor. Phys. 85430

[6] Man'ko O and Man'ko V I 1997 J. Russ. Laser Res. 18407

[7] Man'ko V I and Mendes R V 2000 Physica D 145330

[8] Man'ko V I, Marmo G, Simoni A, Sudarshan E C G and Ventrigilia F 2008 Rep. Math. Phys. 61 337

[9] Ibort A, Man'ko V I, Marmo G, Simoni A and Ventriglia F 2009 Phys. Scr. 79065013

[10] Dodonov V V, Man'ko O V, Man'ko V I and Wünsche A 1999 Phys. Scr. 5981

[11] Wünsche A, Dodonov V V, Man'ko O V and Man'ko V I 2001 Fortschr. Phys. 491117

[12] Man'ko O V, Man'ko V I and Marmo G 2000 Phys. Scr. 62446

[13] Man'ko O V, Man'ko V I and Marmo G 2002 J. Phys. A: Math. Gen. 35699

[14] Man'ko O V, Man'ko V I, Marmo G and Vitale P 2007 Phys. Lett. A 360522

[15] Man'ko V I, Marmo G and Vitale P 2005 Phys. Lett. A 3341

[16] Castaños O, López-Peña R, Man'ko M A and Man'ko V I 2003 J. Phys. A: Math. Gen. 364677

[17] Filippov S N and Man'ko V I 2008 J. Russ. Laser Res. 30129

[18] Filippov S N and Man'ko V I 2008 J. Russ. Laser Res. 30224

[19] Wallentowitz S and Vogel W 1996 Phys. Rev. A $\mathbf{5 3} 4528$

[20] Banaszek K and Wodkiewicz K 1996 Phys. Rev. Lett. 764344

[21] Mancini S, Man'ko V I and Tombesi P 1997 Europhys. Lett. 3779

[22] Man'ko O V and Man'ko V I 2003 J. Russ. Laser Res. 24497

[23] Cahill K E and Glauber R J 1969 Phys. Rev. 1771882

[24] Fuchs C A 1996 Distinguishability and accessible information in quantum theory Preprint quant-ph/9601020 
[25] Nielsen M A and Chuang I L 2000 Quantum Computation and Quantum Information (Cambridge: Cambridge University Press)

[26] Uhlmann A 1976 Rep. Math. Phys. 9273

[27] Jozsa R 1994 J. Mod. Opt. 412315

[28] Heinosaari T and Ziman M 2008 Acta Physica Slovaca 58487

[29] Filippov S N and Man'ko V I 2008 J. Russ. Laser Res. 29564

[30] Bhattacharyya A 1943 Bull. Calcutta Math. Soc. 3599 